\title{
Acute Coronary Syndrome Secondary to Acute Aortic Dissection - Underlying Mechanisms and Possible Therapeutic Options
}

\author{
Kazuhito Hirata ${ }^{1}$, Tomoya Hiratsuji ${ }^{2}$, \\ Minoru Wake ${ }^{1}$ and Hidemitsu Mototake ${ }^{3}$ \\ ${ }^{1}$ Okinawa Chubu Hospital, Division of Cardiology \\ ${ }^{2}$ Okinawa Hokubu Hospital,Division of Cardiology \\ ${ }^{3}$ Okinawa Chubu Hospital, Cardiovascular Surgery \\ Japan
}

\section{Introduction}

Acute coronary syndrome(ACS) and acute aortic dissection(AAD) are life-threatening conditions which can be difficult to differentiate in the emergency room because of the similarity of clinical presentations. In addition, ACS can be caused in AAD as a complication of the dissecting process. Usual form of ACS is caused by an obstruction of the epicardial coronary arteries, which is initiated with the rupture of an unstable atherosclerotic plaque complicated with subsequent thrombus formation(Libby , 2001). On the other hand, ACS secondary to AAD is caused by malperfusion of the coronary artery by an obstruction of the orifice as a complication of the dissecting process. If AAD is compicated with ACS, prognosis becomes worse and the treatment of choice may be totally different from usual ACS. Medications and procedures which are usually used in cases of ordinary ACS, such as Heparin, antiplatelets, thrombolytic agents and catheter interventions, may be harmful in ACS secondary to AAD. So it is of great importance to make a correct diagnosis of ACS secondary to AAD for better treatment and survival.

\section{Acute coronary syndrome in patients with acute type $A$ aortic dissection}

\subsection{Incidence}

Incidence of coronary involvement in AAD has been reported to be 1.8-11.3\%(Hirst, 1958 ; Hagan, 2000 ; Kawahito, 2003 ; Neri, 2001 ; Spittel, 1993). Incidence may vary according to the different study population(autopsy, surgical patients or non-selected patients in the emergency room). We recently evaluated the incidence of coronary malperfusion in 159 patients with type A AAD who presented to the emergency room within 12 hours from the onset and found that $9.4 \%$ had coronary malperfusion(Hirata, 2010). On the contrary, the incidence of the AAD in ACS is not so frequent. In the large studies dealing with prehospital thrombolysis, $0.3-0.33 \%$ of the patient was erroneously diagnosed as having usual ACS who later proved to have AAD(Wilcox,1988; European Myocardial Infarction Group, 1993). 


\subsection{Mechanisms}

There are four possible mechanisms for coronary malperfusion in $\mathrm{AAD}$ (Ashida, 2000 ; Cambria, 1988; Massetti, 2003 ; Neri, 2001 ; Shapira, 1998,)

1. buldging of the dissected false lumen producing occlusion of the coronary artery orifice (Figure $1 \mathrm{~A}$ ).

2. a retrograde extension of the dissection into the coronary arterial wall resulting in obstruction (Figure $1 \mathrm{~B}$ )

3. disruption or detachment of the coronary artery from the aortic $\operatorname{root}($ Figure $1 \mathrm{C}$ ).

4. dynamic obstruction of the coronary orifice by flail intimal flap(Figure $1 \mathrm{D}$ ).
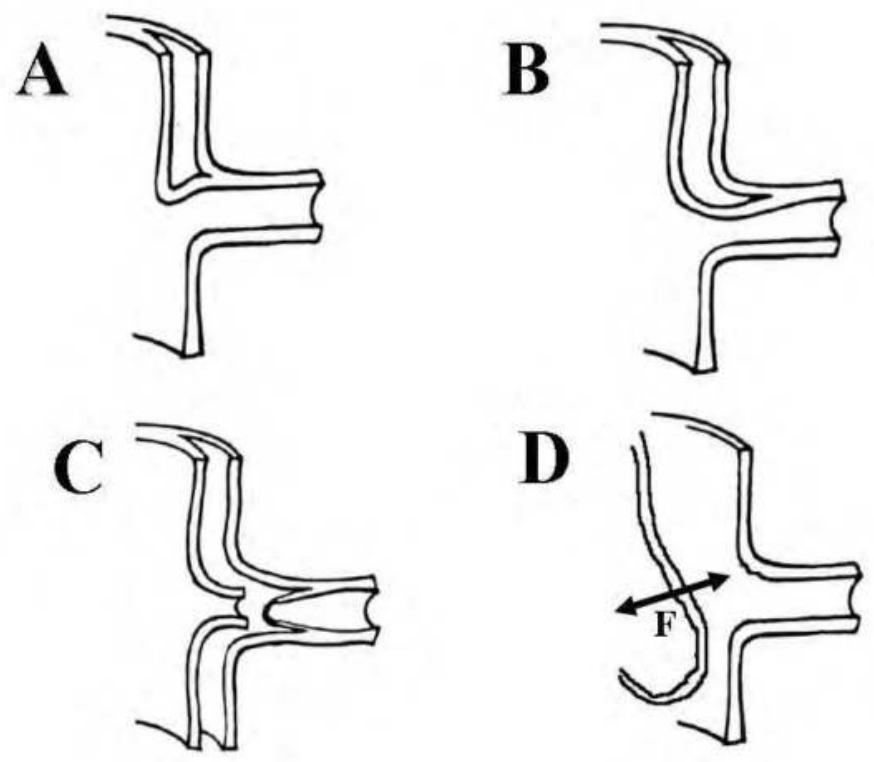

Fig. 1. Four possible mechanisms for coronary malperfusion in cases of acute type A aortic dissection. Mechanisms A to D correspond to mechanisms 1) to 4) in the text. See text in detail (modified from Neri E et al. Proximal aortic dissection with coronary artery malperfusion. J Thorac Cardiovasc Surg 2001 ; 121 : 552-560 with permission).

Distribution of the each mechanism has not been well defined. In our experience (Hirata,2010), among 10 patients in whom actual mechanism of coronary malperfusion was identified during surgery, 5 patients had mechanism 1), 3 patients had mechanism 2), and 2 patients had mechanism 3). Identification of the mechanism for coronary malperfusion is potentially important in relation to the therapeutic options described later.

\subsection{Clinical pictures and electrocardiographic changes}

Compared with patients of AAD without ACS, those with ACS had more severe clinical presentation as manifested with a higher incidence of cardiac tamponade and initial shock vital signs(Hirata K, 2010). The prognosis was also worse(Kawahito,2003; Metha 2002). If the right coronary artery is involved, ST segment elevation in the inferior leads 
is seen. Simultaneous ST elevation in leads V1-V3 may be seen as a result of obstruction of the conus branch or the right ventricular branch (Figure 2). If the left main trunk(LMT) is involved, elevation of the ST segment in leads aVR and aVL with diffusse ST depression in other leads(Figure 3), or ST elevation in V1-V6 and I, aVL are seen(Nikus,2007).
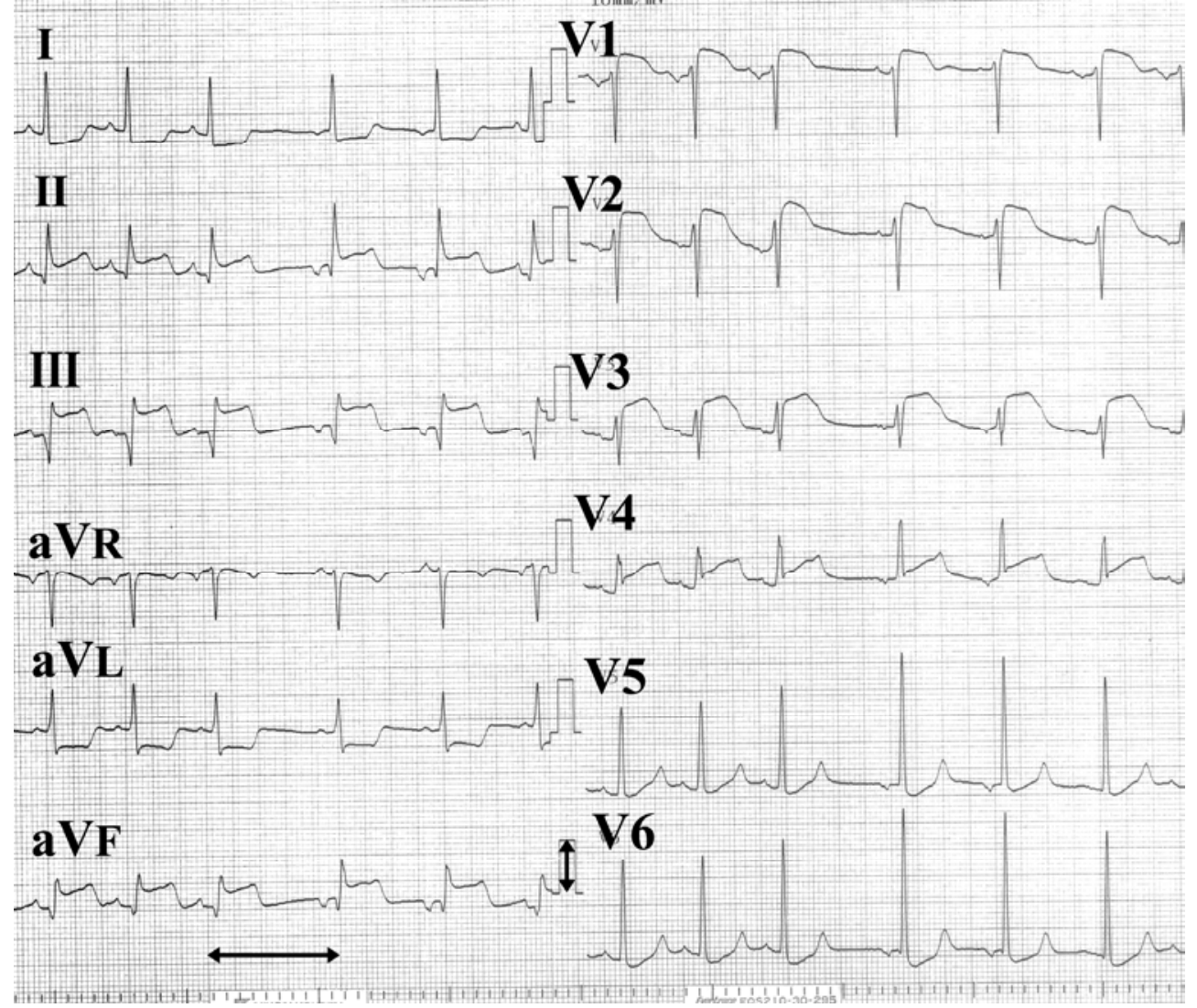

Fig. 2. Twelve-lead ECGs obtained from a 56-year-old male with type A AAD. This patient had shock (initial systolic blood pressure was $60 \mathrm{mmHg}$ ), cardiac tamponade and mild aortic regurgitation. An ECG showed sinus pause with ectopic atrial escape rhythm, marked ST elevation in both inferior and precordial leads. This patient had disruption of the orifice of right coronary artery but the orifice of left main trunk was intact. Simultaneous ST elevation in inferior and anterior leads reflected involvement of the conus branch or right ventricular branch due to obstruction of the orifice of the right coronary artery. A horizontal arrow indicates 1 second and a vertical arrow indicates $1 \mathrm{mV}$ (same in Figure 3). Reproduced with permission(Hirata K et al. Electrocardiographic changes in patients with type A acute aortic dissection. J Cardiol 2010;56:147-153). 


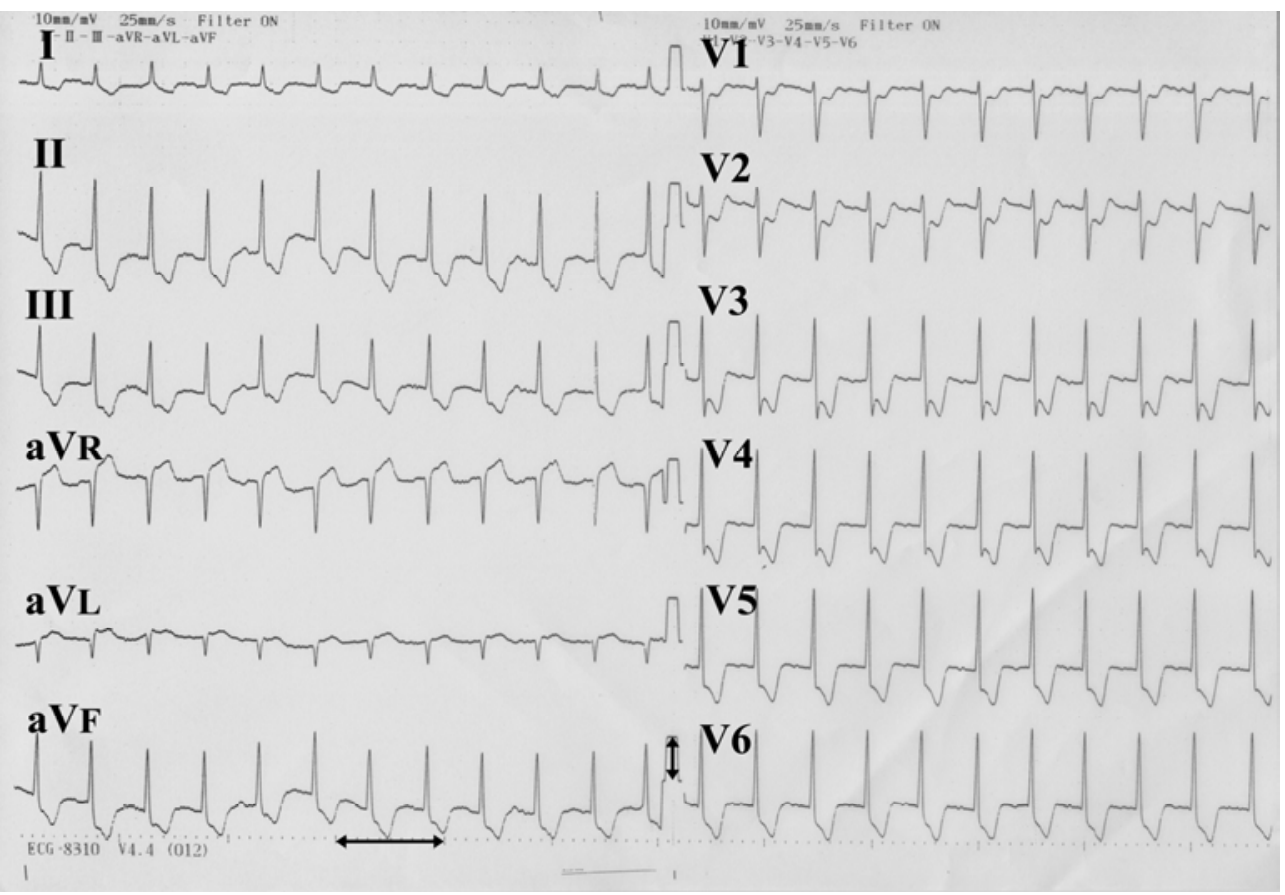

Fig. 3. Twelve-lead ECGs obtained from a 46-year-old female with type A AAD associated with Marfan syndrome. Note that ST segment was elevated in leads aVR and aVL, and diffuse severe ST depression was also seen. This patient had acute pulmonary edema due to severe acute aortic valvular regurgitation. Initial systolic blood pressure was preserved $(120 \mathrm{mmHg})$. A transesophageal echocardiogram showed flail intimal flap in the ascending aorta. The dissection was extended beyond the orifice of the left main trunk. Bentall surgery and coronary reconstruction were performed. Reproduced with permission(Hirata K et al. Electrocardiographic changes in patients with type A acute aortic dissection. J Cardiol 2010;56:147-153).

We recently reported that acute electrocardiographic changes(either ST depression and or T wave inversion) were rather common in patients with type A AAD even if there was no involvement of the coronary artery(Table 1, Hirata 2010). Only $27 \%$ of the patients with type A AAD had normal ECG. These observations were consistent with others(Hagan, 2000). Those acute ECG changes were closely associated with initial shock state $(\mathrm{BP}<90 \mathrm{mmHg})$ and cardiac tamponade in our experience of 159 patients(Hirata K, 2010).

Pre-existing chronic coronary artery disease has been reported to coincide in some patients with type A AAD. In the international registry of AAD, Hagan et al reported that, among 464 patients, $4.3 \%$ had previous history of bypass surgery and $7.7 \%$ had evidence of old myocardial infarction(Hagan, 2000). Creswell et al. found that about one-third of the patients showed one or more coronary artery lesions greater than $50 \%$ with a coronary angiography (Creswell, 1995). At present, in type A AAD, the contribution of preexisting chronic coronary disease on clinical presentation, acute ECG change and needs for concomitant bypass surgery has not been well defined. 
Chronic ECG abnormalities such as left ventricular hypertrophy with or without strain pattern, bundle branch block, etc. were also common in patients with type A AAD.

\begin{tabular}{cl}
\hline Acute change & $79(49.7)$ \\
ST elevation $(\geqq 0.1 \mathrm{mV})$ & $13(8.2)$ \\
ST depression & $54(34.0)$ \\
$\geqq 0.1 \mathrm{mV}$ and $<0.2 \mathrm{mV}$ & $28(17.6)$ \\
$\geqq 0.2 \mathrm{mV}$ and $<0.3 \mathrm{mV}$ & $18(11.3)$ \\
$\geqq 0.3 \mathrm{mV}$ & $8(5.0)$ \\
T inversion & $34(21.4)$ \\
AVB & $3(1.9)$ \\
New Af & $1(0.6)$ \\
PAC/PVC & $5(3.1)$ \\
Sinus bradycardia & $18(11.3)$ \\
Chronic change & $58(36.5)$ \\
LVH with strain & $15(9.4)$ \\
LVH voltage & $17(10.7)$ \\
Q waves & $6(3.8)$ \\
BBB & $8(5.0)$ \\
Chronic Af & $8(5.0)$ \\
Both acute and chronic & $21(13.2)$ \\
Normal & $43(27.0)$ \\
\hline
\end{tabular}

AVB: Atrioventricular block ( $\geqq$ second degree), Af: Atrial fibrillation, PAC: Premature Atrial Contraction, PVC: Premature Ventricular Contraction, LVH: Left Ventricular Hypertrophy, BBB : Bundle Branch Block, Values are expressed as number(\%). Reproduced with permission(Hirata K et al. Electrocardiographic changes in patients with type A acute aortic dissection. J Cardiol 2010;56:147-153)

Table 1. ECG changes in type A AAD $(n=159)$

\subsection{Differentiation between acute coronary syndrome secondary to acute type A aortic dissection and usual form of acute coronary syndrome}

Regardless of the difference of the mechanism, the results of coronary malperfusion is myocardial ischemia in both usual form of ACS and ACS secondary to AAD. So it is impossible to differentiate usual form of ACS from ACS secondary to AAD with electrocardiographic findings. At present, differential diagnosis is dependent on the clinical suspicion based on difference of clinical pictures, confirmed with imaging modalities such as a CT scan and an echocardiogram. Shirakabe et al. tried to differentiate AAD and ACS using scoring system utilizing clinical idexes obtainable in emergency room. Presence of back pain, mediastinal widening on chest X-ray, aortic regurgitation and aortic dilatation $(>30 \mathrm{~mm})$ were closely associated with AAD (Shirakabe,2008). They found that AAD can be differentiated from ACS at sensitivity of $93.1 \%$ and specificity of $77.6 \%$, when more than 3 of the 4 features were positive. We found that ECG change in AAD is closely associated with initial shock vital sign $(\mathrm{BP}<90 \mathrm{mmHg})$ and cardiac tamponade (Hirata, 2010). To perform a trans-thoracic echocardiography at bedside in the emergency room appeared to be very important in ACS 
patients in whom underlying AAD is a possible cause of ACS. Those patients include abrupt onset with back pain, shock vital signs, mediastinal widening(ratio of greater than $30 \%$ ), pericardial effusion, aortic regurgitation, and aortic root dilatation $(>30 \mathrm{~mm})$. If there is a flail intimal flap, the diagnosis of AAD can be made at bedside

\section{Treatment options for acute coronary syndrome secondary to acute type A aortic dissection}

\subsection{Surgery}

If ACS secondary to AAD is erroneously diagnosed as usual form of ACS and received thrombolysis or catheter interventions, those patients may have an increased risk of developing harmful sequelae such as rupture of the aorta resulting in bleeding or cardiac tamponade (Blankenship, 1989; Butler 1990; Eriksen, 1992; Kamp,1994; Melchior,1993). Surgical treatment to improve coronary malperfusion and underlying dissecting process at the same time is the most important and vital(Kawahito,2003; Neri,2001). Replacement of the dissected ascending aorta(sometimes beyond the arch) with artificial vessel prosthesis is necessary. If coronary involvement is seen, simultaneous bypass surgery or repair of the involved coronary artery may be required. Especially, if coronary malperfusion is due to mechanism 2) and 3), bypass surgery is mandatory(Figure 1 B and C). In mechanism 1), decompression of the false lumen may be sufficient enough to restore coronary perfusion(Figure 1A). In mechanism 4), removal of the flap with ascending aortic replacement may be effective and bypass may not be necessary(Figure1 D). Needless to say, aortic valve replacement is necessary, if the aortic valve regurgitation is severe.

\subsection{Percutaneous catheter intervention}

There have been several case reports of successful percutaneous catheter intervention (PCI) for ACS secondary to AAD. Barabas et al. reported a case of intermittent obstruction of the LMT in whom deployment of a stent in LMT was very effective to improve unstable hemodynamic (Barabas,2000). The patient was later sent to surgery as a definitive treatment. In their case, the diagnosis of ACS secondary to AAD was not made before the catheter intervention. The presence of AAD was diagnosed during the procedure and unplanned stenting was performed as an emergency bridge to surgery. Yunoki et al. reported a rare case of type A AAD in whom the right coronary orifice obstruction resulted in acute inferior and right ventricular myocardial infarction(Yunoki, 2010). Initially, the patient was sent for catheter intervention as having an usual form of ACS. During the procedure, AAD was diagnosed and the stent was deployed at the orifice of the right coronary artery. The patient was carefully followed medically and at one year, a false lumen in the ascending aorta had been resolved.

\subsubsection{Report of a case}

We recently experienced a case of ACS secondary to AAD in whom, emergency stenting in the LMT was intentionally necessary. The patient was a 56-year-old female who presented to the emergency room of Okinawa Hokubu Hospital complaining of chest pain. Initial systolic blood pressure was $60 \mathrm{mmHg}$ and heart rate was 80 beats per minutes. A chest $\mathrm{X}$ ray showed mediastinal widening. A bedside echocardiography and a CT scan confirmed type A AAD(Figure 4). ECG showed ST elevation in aVR, aVL and V5-6. The patient 
developed repetitive episodes of ventricular fibrillation requiring multiple cardioversion and cardiopulmonary resuscitation. The patient was intubated and was taken to the catheterization laboratory. A percutaneous cardiopulmonary support equipment(PCPS) was inserted and emergency catheter intervention was performed as a last resort, because the hospital was not equipped for emergency cardiovascular surgery. A coronary angiography showed obstruction of the LMT with a dissecting hematoma(Figure 5). A bare metal stent was deployed at LMT. Shortly after, the patient's hemodynamic condition improved and the patient was transferred to Okinawa Chubu Hospital (with a support of PCPS). Emergency surgery (CABG on LAD and ascending aortic replacement) was performed. At the time of surgery, the surgeons noticed the ischemia of the small bowel and the part of the small bowel was also resected. The patient's condition improved temporarily, but unfortunately the patient died of pan-peritonitis secondary to intestinal ischemia and necrosis. In this particular patient, emergency left main stenting was the last resort in the situation of very unstable hemodynamic condition and inaccessibility of on-site cardiac surgery.
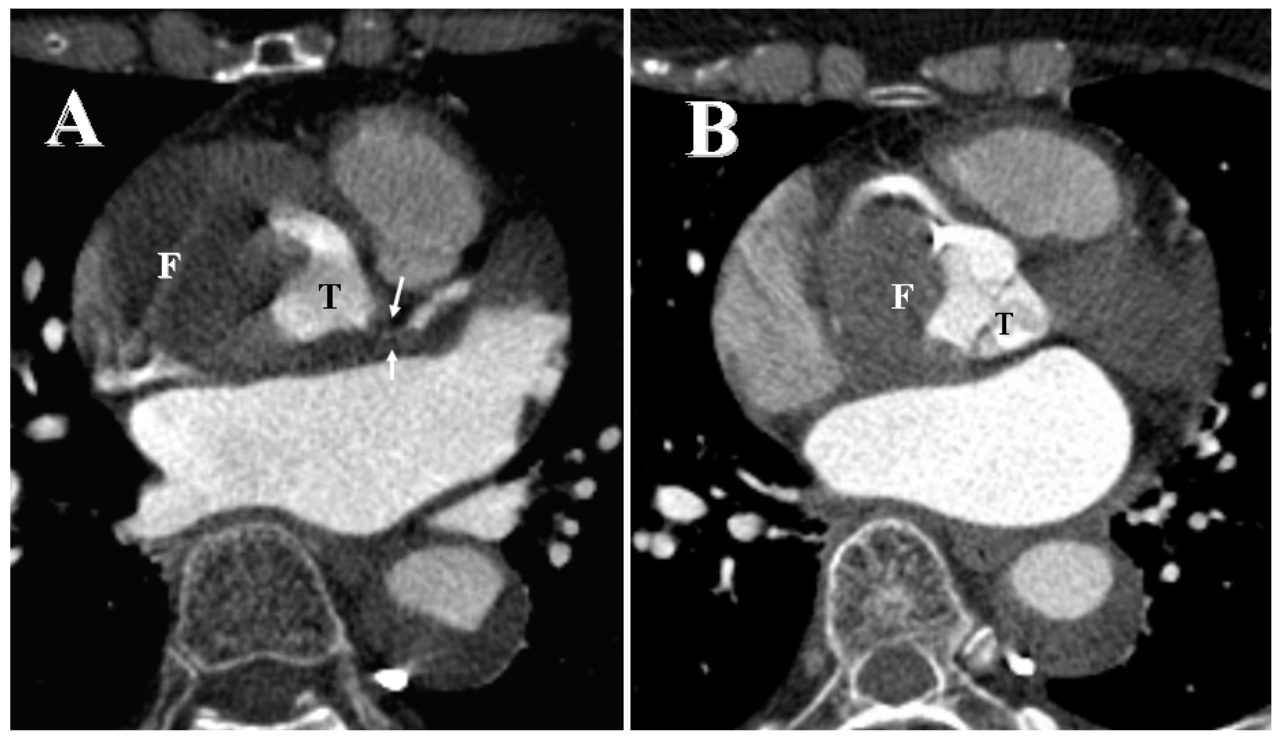

Fig. 4. A: A CT scan image showing severe narrowing of the left main coronary artey (between arrows)due to the extension of the dissecting hematoma. B: The ostium of the right coronary artery was not obstructed. $\mathrm{T}$ : True lumen, F: False lumen. 


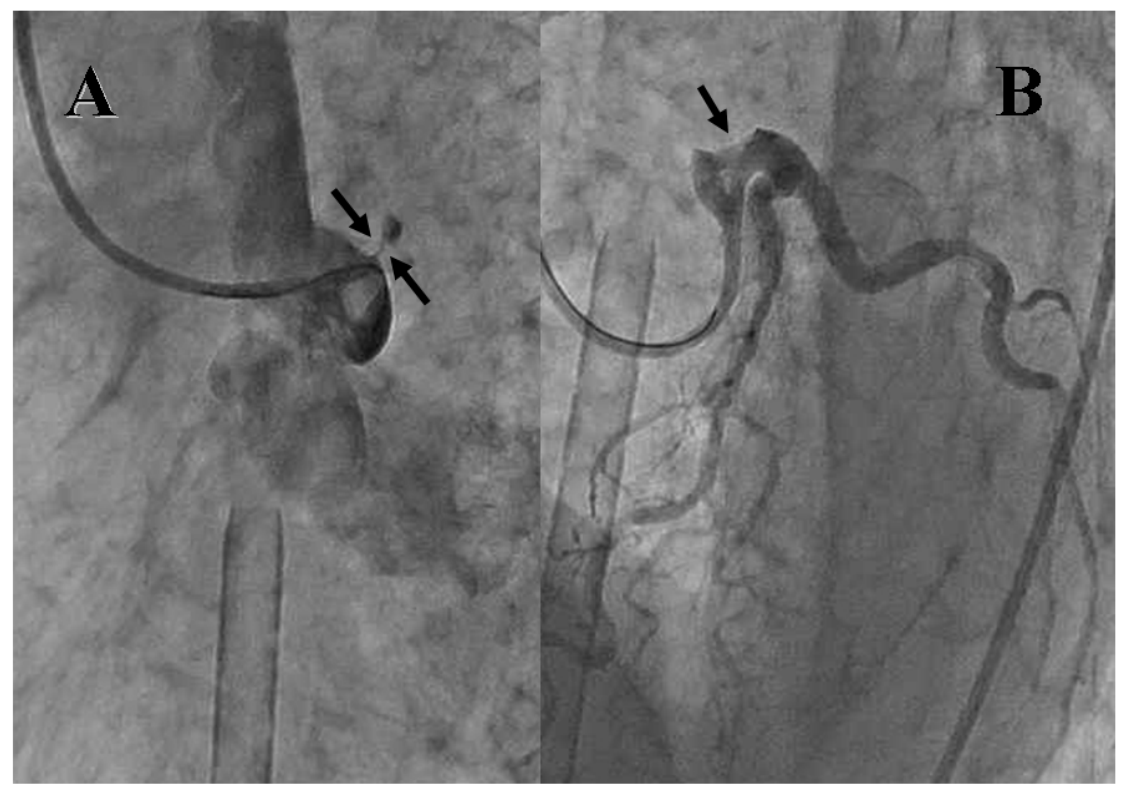

Fig. 5. Coronary angiograms before(A) and after(B) stenting the left main coronary artery. Note the severe narrowing of the orifice of the left main coronary artery resulting in poor visualization of both left anterior descending and circumflex artery. After stenting the left main tract became wide open and TIMI 3 flow was restored in both left anterior descending and circumflex arteries. A:Left anterior oblique view. B:Left anterior oblique and cranial view.

\subsubsection{Current shortcomings of catheter intervention}

Current shortcomings of PCI for ACS secondary to AAD include the followings:

1. Although PCI may be temporarily effective to improve coronary malperfusion, it has no effects on the ongoing dissection process itself. So the surgical approach to the dissecting process may be inevitably necessary

2. Medications used after PCI, such as antiplatelets or heparin, may result in increased risk of bleeding, rupture or cardiac tamponade.

3. To insert and to advance the guiding catheter in the injured aortic lumen may result in further injury of the aortic wall(sometimes, the true lumen is so shrunk and deformed). In addition, the guiding catheter may be erroneously inserted into the false lumen

4. Intra-aortic balloon pumping may be contraindicated and the safety of PCPS is not established.

5. If the mechanism of coronary malperfusion is due to mechanism 3), to pass the guidewire across the obstruction is impossible because there is no continuity of the wall of the coronary artery(Figure $1 \mathrm{C}$ ).

6. If the malperfusion is due to mechanism 4), deploying a stent in the orifice is not effective(Figure 1D).

So, at present, ACS secondary to AAD should be treated with surgery in most cases. PCI may be effective in selected cases as a bridge to definitive surgery. 


\section{Conclusion}

Recognition and appropriate management of ASC secondary to AAD is very important. Because the mechanism of coronary malperfusion is totally different from usual ACS, the treatment of choice is also very different. Making a correct diagnosis regarding underlying dissection is very important.

\section{Acknowledgment}

We deeply express our thanks to Drs. Maeshiro, Henzan, Ie, Tengan, Yasumoto and Asato. We also appreciate the hard work of all the past and current fellow doctors in the division of cardiology, in Okinawa Hokubu and Chubu Hospital

\section{References}

Ashida K. (2000). A case of aortic dissection with transient ST-segment elevation due to functional left main coronary arterial obstruction. Jpn Circ J ,64,130-4

Barabas M.(2000). Left Main Stenting-as a Bridge to Surgery- for Acute Type A Aortic dissection and Anterior Myocardial Infarction. Catheter Cardiovasc Intervnt, 51, 7477.

Blankenship J.(1989). Cardiovascular complications of thrombolytic therapy in patients with a mistaken diagnosis of acute myocardial infarction. J Am Coll Cardiol, 15 ,1579-82.

Butler J.(1990). Streptokinase in acute aortic dissection. Br Med J 1990, 300, 517-9.

Creswell LL. (1995). Coronary artery disease in patients with type A aortic dissection. Ann Thorac Surg , 59 , 585-90.

Eriksen UH(1992). Fatal haemostatic complications due to thrombolytic therapy in patients falsely diagnosed as acute myocardial infarction. Eur Heart $J, 13,840-3$.

Hagen PG.(2000). The international registry of acute aortic dissection(IRAD) : New insight into an old disease. JAMA, 283, 897-903.

Hirata K.(2010). Electrocardiographic changes in patients with type A acute aortic dissection-incidence, patterns and underlying mechanisms in 159 cases. J Cardiol, 56 , 147-153.

Kamp TJ.(1994) Myocardial infarction, aortic dissection, and thrombolytic therapy Am Heart $J, 128,1234-7$.

Libby P.(2001). Current concept of the pathogenesis of acute coronary syndromes Circulation $, 104,365-372$

Maseetti M.(2003). Flap suffocation : An uncommon mechanism ofcoronary malperfusion in acute type A dissection. J Thrac Cardiovasc Surg, 125, 6, 1548-1550.

Metha RH. (2002). Predicting death in patients with acute type A aortic dissection. Circulation, 105, 200-2006

Melchior T.(1993) Aortic dissection in the thrombolyitic era : early recognition and optimal management is a prerequisite for improved survival. Int J Cardiol 1993,42, 1-6.

Neri E.(2001). Proximal aortic dissection with coronary malperfusion: presentation, management and outcome. J Thrac Cardivasc Surg ,121, 552-60 .

Nikus KC.(2007). Acute total occlusion of left main coronary artery with emphasis on electrocardiographic manifestations. Timely Top Med Cardiovasc Dis , 11 , E22. 
Shapira OM.(1998). Functional left main coronary artery obstruction due to aortic dissection. Circulation, 98,278-80.

Shirakabe A.(2008). Diagnostic score to differenciate acute aortic dissection in the emergency room. Circ J, 72, 986-990.

Spittel PC.(1993). Clinical features and differential diagnosis of aortic dissection :experience with 236 cases(1986 through 1990). Mayo Clin Proc. , 68 , 642-51.

The European Myocardial Infarction Project Group.(1993) Prehospital thrombolysis in patients with suspectedacute myocardial infarction. N Engl J Med , 329 ,383-9.

Wilcox RG.(1988) Trial of tissue plasminogen activator for mortality reduction in acute myocardial infarction. Anglo-Scandinavian Study of Early Thrombolysis(ASSET). Lancet , 2 , 525-30.

Yunoki K.(2010). Stenting of right coronary ostial occlusion due to thrombosed type A aortic dissection : One-year follow-up results. J Cardiol case , 1, e116-170. 


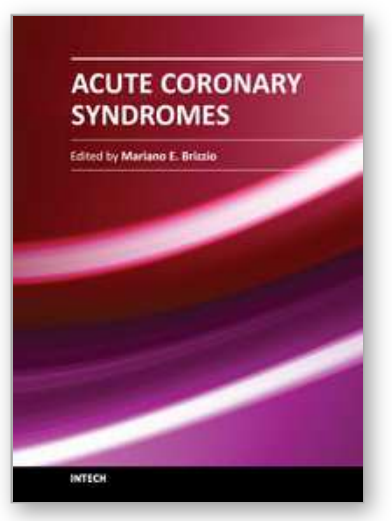

\author{
Acute Coronary Syndromes \\ Edited by Dr. Mariano Brizzio
}

ISBN 978-953-307-827-4

Hard cover, 214 pages

Publisher InTech

Published online 24, February, 2012

Published in print edition February, 2012

This book has been written with the intention of providing an up-to-the minute review of acute coronary syndromes. Atherosclerotic coronary disease is still a leading cause of death within developed countries and not surprisingly, is significantly rising in others. Over the past decade the treatment of these syndromes has changed dramatically. The introduction of novel therapies has impacted the outcomes and surviving rates in such a way that the medical community need to be up to date almost on a "daily bases". It is hoped that this book will provide a timely update on acute coronary syndromes and prove to be an invaluable resource for practitioners seeking new and innovative ways to deliver the best possible care to their patients.

\title{
How to reference
}

In order to correctly reference this scholarly work, feel free to copy and paste the following:

Kazuhito Hirata, Tomoya Hiratsuji, Minoru Wake and Hidemitsu Mototake (2012). Acute Coronary Syndrome Secondary to Acute Aortic Dissection - Underlying Mechanisms and Possible Therapeutic Options, Acute Coronary Syndromes, Dr. Mariano Brizzio (Ed.), ISBN: 978-953-307-827-4, InTech, Available from:

http://www.intechopen.com/books/acute-coronary-syndromes/acute-coronary-syndrome-secondary-to-acuteaortic-dissection

\section{INTECH}

open science | open minds

\section{InTech Europe}

University Campus STeP Ri

Slavka Krautzeka 83/A

51000 Rijeka, Croatia

Phone: +385 (51) 770447

Fax: +385 (51) 686166

www.intechopen.com

\section{InTech China}

Unit 405, Office Block, Hotel Equatorial Shanghai

No.65, Yan An Road (West), Shanghai, 200040, China 中国上海市延安西路65号上海国际贵都大饭店办公楼 405 单元

Phone: +86-21-62489820

Fax: +86-21-62489821 
(C) 2012 The Author(s). Licensee IntechOpen. This is an open access article distributed under the terms of the Creative Commons Attribution 3.0 License, which permits unrestricted use, distribution, and reproduction in any medium, provided the original work is properly cited. 